\title{
Energy storage and dispersion of surface acoustic waves trapped in a periodic array of mechanical resonators
}

\author{
Dühring, Maria Bayard; Laude, Vincent; Khelif, Abdelkrim
}

Published in:

Journal of Applied Physics

Link to article, DOI:

$10.1063 / 1.3114543$

Publication date:

2009

Document Version

Publisher's PDF, also known as Version of record

Link back to DTU Orbit

Citation (APA):

Dühring, M. B., Laude, V., \& Khelif, A. (2009). Energy storage and dispersion of surface acoustic waves trapped in a periodic array of mechanical resonators. Journal of Applied Physics, 105(9), 093504.

https://doi.org/10.1063/1.3114543

\section{General rights}

Copyright and moral rights for the publications made accessible in the public portal are retained by the authors and/or other copyright owners and it is a condition of accessing publications that users recognise and abide by the legal requirements associated with these rights.

- Users may download and print one copy of any publication from the public portal for the purpose of private study or research.

- You may not further distribute the material or use it for any profit-making activity or commercial gain

- You may freely distribute the URL identifying the publication in the public portal 


\title{
Energy storage and dispersion of surface acoustic waves trapped in a periodic array of mechanical resonators
}

\author{
Maria B. Dühring, ${ }^{1, a)}$ Vincent Laude, ${ }^{2}$ and Abdelkrim Khelif ${ }^{2}$ \\ ${ }^{1}$ Department of Mechanical Engineering, Solid Mechanics, Technical University of Denmark, 2800 Lyngby, \\ Denmark \\ ${ }^{2}$ Institut FEMTO-ST, Université de Franche-Comté, CNRS, ENSMM, UTBM, 32 avenue de l'Observatoire, \\ F-25044 Besançon, France
}

(Received 12 January 2009; accepted 25 February 2009; published online 4 May 2009)

\begin{abstract}
It has been shown previously that surface acoustic waves can be efficiently trapped and slowed by steep ridges on a piezoelectric substrate, giving rise to two families of shear-horizontal and vertically polarized surface waves. The mechanisms of energy storage and dispersion are explored by using the finite element method to model surface acoustic waves generated by high aspect ratio electrodes. A periodic model is proposed including a perfectly matched layer to simulate radiation conditions away from the sources, from which the modal distributions are found. The ratio of the mechanical energy confined to the electrode as compared to the total mechanical energy is calculated and is found to be increasing for increasing aspect ratio and to tend to a definite limit for the two families of surface waves. This observation is in support of the interpretation that high aspect ratio electrodes act as resonators storing mechanical energy. These resonators are evanescently coupled by the surface. The dispersion diagram is presented and shows very low group velocities as the wave vector approaches the limit of the first Brillouin zone. (C) 2009 American Institute of Physics. [DOI: 10.1063/1.3114543]
\end{abstract}

\section{INTRODUCTION}

Surface acoustic wave (SAW) transduction by the use of interdigital transducers ${ }^{1}$ (IDTs) is a widespread technique for the electrical transduction in piezoelectric materials. SAWs are elastic waves that propagate along a material surface with most of their energy density concentrated at the surface. ${ }^{2}$ In most practical cases, for example, for the design of classical resonators, filters, and sensors, ${ }^{3-5}$ only the electrical properties of the IDT are of interest. In these applications SAWs are generated by IDTs with limited electrode height such that the SAW mode properties do not differ appreciably from those of a free or a fully metallized surface, except for the periodic aspects of wave propagation. For instance, propagation remains only slightly dispersive and the polarization of surface waves is relatively unaltered. In Ref. 6, however, it was shown theoretically by a finite element method (FEM)/ boundary element method algorithm that the SAW properties are significantly changed when high aspect ratio (HAR) electrodes are used instead. Multimode SAW propagation was found and up to a tenfold slowing of the SAW phase velocity was obtained. These results were confirmed by experiments. ${ }^{7}$ Recently, related work has been performed using the finite element and finite difference time domain method as well as experiments to show how band gaps and resonances change for increasing stub height in a plate with a periodic stubbed surface. $^{8,9}$

The FEM/boundary element method is widely used for obtaining the harmonic admittance ${ }^{10}$ as well as the harmonic displacements under the electrodes of a periodic infinite array of electrodes on a piezoelectic substrate..$^{6,11,12}$ An alter-

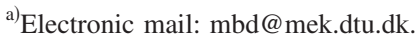

native method is to use directly the FEM with appropriate periodic boundary conditions. ${ }^{13}$ The difficulty in this case, however, is to account for the semi-infinite nature of the substrate, which can result in oversized meshes and then impractical calculations. Here we introduce a full finite element model of a piezoelectric, anisotropic material to simulate the HAR electrodes utilizing periodic boundary conditions. A perfectly matched layer (PML) is employed at the bottom to absorb the acoustic and electric disturbances propagating away from the surface ${ }^{14-17}$ With this model it is possible to obtain the mode shape of all the resonances for the periodic structure such that it can be explained how the vibration of the surface interacts with the electrode for the different modes. We then use the model to calculate the mechanical energy stored in the electrode compared to the mechanical energy in the total structure in order to explain why the phase velocity is slowed down for increasing electrode aspect ratio. Finally, the dispersion and group velocity of the different modes are reported.

\section{THE ACOUSTIC MODEL}

A two dimensional (2D) periodic model of an electrode array on the surface of a piezoelectric material is used. The unit cell and the computational domain are illustrated in Fig. 1. The electrode consists of nickel $(\mathrm{Ni})$ and is placed on a substrate of $\mathrm{Y}+128$ cut lithium niobate, $\mathrm{LiNbO}_{3}$. Material constants for lithium niobate are taken from Ref. 18, and for nickel, Young's modulus $E=200 \mathrm{GPa}$ and Poisson's ratio $\nu$ $=0.31$ are used. The width of the electrode is $a$ and the height is $h$. The parameter $p=2 a$ is the acoustic period in the problem and is of the order of a few microns in applications. The SAW is generated by applying an alternating electrical 


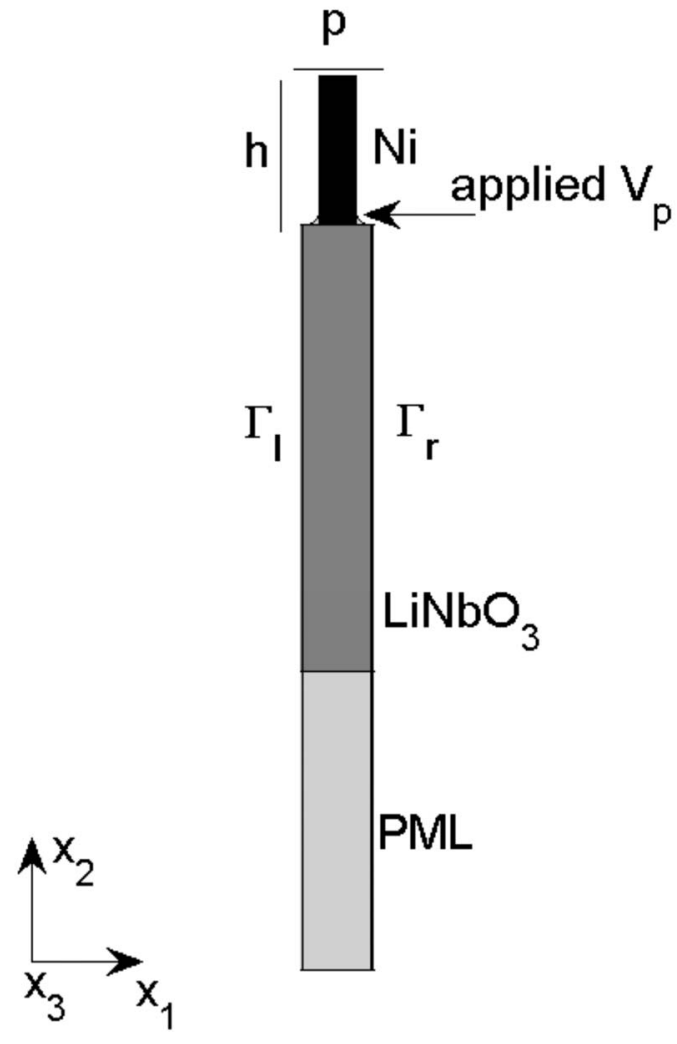

FIG. 1. The geometry of the unit cell with periodic boundary conditions and period $p$. The electrode consists of nickel and has the height $h$. The substrate is lithium niobate and a PML is placed at the bottom. The electric potential $V_{p}$ is applied at the interface between the electrode and the substrate.

potential to the HAR electrodes. This is modeled by applying a positive potential to the electrode in the unit cell and then using periodic boundaries with opposite sign. As the metal is supposed to be perfectly conducting, the electrical potential needs only to be applied at the interface between $\mathrm{Ni}$ and $\mathrm{LiNbO}_{3}$. To prevent reflections of the SAW from the bottom, a PML (Ref. 14) is applied as illustrated on Fig. 1. In Ref. 15, PMLs are introduced for time-harmonic elastodynamic problems and are extended to piezoelectric materials in Refs. 16 and 17. The PMLs have the property that the mechanical and electrical disturbances are gradually absorbed in the layers before they reach the outer boundaries. In this way there are no reflections that can disturb the propagation of the SAW.

The applied electric potential will introduce mechanical deformations in the solid by the inverse piezoelectric effect and the behavior of the piezoelectric material is described by the following model as found in Ref. 19. A time-harmonic electric potential

$$
V\left(x_{j}, t\right)=V\left(x_{j}\right) e^{i \omega t},
$$

with the angular frequency $\omega$, is applied to the electrode. The mechanical strain $S_{i j}$ and the electric field $E_{j}$ are given by the expressions

$$
S_{i j}=\frac{1}{2}\left(\frac{1}{\gamma_{j}} \frac{\partial u_{i}}{\partial x_{j}}+\frac{1}{\gamma_{i}} \frac{\partial u_{j}}{\partial x_{i}}\right) \quad \text { and } \quad E_{j}=-\frac{1}{\gamma_{j}} \frac{\partial V}{\partial x_{j}},
$$

where $u_{i}$ are the displacements and $x_{i}$ are the coordinates. Note that the Einstein notation is not applied in the expressions in Eq. (2). The parameter $\gamma_{j}$ is an artificial damping at position $x_{j}$ in the PML. As the PML is added at the bottom of the structure only $\gamma_{2}$ is different from 1 and is given by the expression

$$
\gamma_{2}\left(x_{2}\right)=1-i \sigma_{2}\left(x_{2}-x_{l}\right)^{2},
$$

where $x_{l}$ is the coordinate at the interface between the regular domain and the PML and $\sigma_{2}$ is a suitable constant. There is no damping outside the PMLs and here $\gamma_{2}=1$. A suitable thickness of the PML as well as the value of $\sigma_{2}$ must be found by calculations such that both the mechanical and electrical disturbances are absorbed before reaching the outer boundaries. However, the absorption must also be sufficiently slow as reflections will occur at the interface between the regular domain and the PML if their material properties are not comparable. The mechanical stresses $T_{j k}$ and the electric displacement $D_{i}$ both depend on the strain and the electric field according to the constitutive relations

$$
\begin{aligned}
& T_{j k}=\widetilde{c}_{j k l m}^{E} S_{l m}-\widetilde{e}_{i j k}^{T} E_{i}, \\
& D_{i}=\widetilde{e}_{i j k} S_{j k}+\widetilde{\varepsilon}_{i j}^{S} E_{j},
\end{aligned}
$$

where $\widetilde{c}_{j k l m}^{E}$ are the elastic stiffness constants, $\widetilde{e}_{i j k}$ are the piezoelectric stress constants, and $\widetilde{\varepsilon}_{i j}^{S}$ are the permittivity constants. The materials are, in general, anisotropic, and as it is only possible to generate the SAW by the inverse piezoelectric effect in certain directions the material tensors have to be rotated. This is indicated by the tilde above the material tensors. The rotation is done according to Euler's transformation theory as explained in Ref. 20. Here the relation between the original directional vector $r_{j}$ and the rotated vector $\hat{r}_{i}$ is given by

$$
\hat{r}_{i}=a_{i j} r_{j},
$$

where $a_{i j}$ is the transformation matrix given by the Euler angles $\varphi_{1}, \varphi_{2}$, and $\varphi_{3}$, where the crystal axes are rotated clockwise about the $x_{3}$-axis, then the $x_{2}$-axis, and finally the $x_{3}$-axis again. The material property matrices can then be transformed by the transformation matrix $a_{i j}$ and the Bond stress transformation matrix $M_{i j m n}$ (the derivation procedure of $M_{i j m n}$ is defined in Ref. 20) as follows:

$$
\begin{aligned}
& \widetilde{c}_{i j k l}^{E}=M_{i j m n} M_{k l p q} c_{m n p q}^{E}, \quad \tilde{e}_{i j k}=a_{i l} M_{j k m n} e_{l m n} \quad \text { and } \\
& \widetilde{\varepsilon}_{i j}^{S}=a_{i k} a_{j l} \varepsilon_{k l}^{S} .
\end{aligned}
$$

The governing equations give the stresses by Newton's second law and the electric displacement from Gauss law,

$$
\frac{1}{\gamma_{j}} \frac{\partial T_{i j}}{\partial x_{j}}=-\rho \omega^{2} u_{i} \quad \text { and } \quad \frac{1}{\gamma_{j}} \frac{\partial D_{i}}{\partial x_{j}}=0
$$

where $\rho$ is the density of the material. Note again that the Einstein notation is not applied in Eq. (8). 
Both mechanical and electrical boundary conditions must be specified to solve the problem. Considering the mechanical conditions, the upper surface is stress-free and the bottom is clamped,

streess free surface: $T_{j k} m_{k}=0$,

clamped surface: $u_{i}=0$,

where $m_{k}$ is the normal unit vector pointing out of the surface. At the upper surface, there are no charges and therefore electric insulation occurs, meaning that the normal component of the electric displacement is zero. At the bottom of the domain it is assumed that the electric potential is zero, whereas at the interface between the electrodes and the substrate the potential is $V_{p}$. The electrical boundary conditions are summarized as follows:

electrical insulation: $D_{i} m_{i}=0$,

zero potential: $\quad V=0$,

applied positive potential: $V=V_{p}$.

Periodic boundary conditions must be induced for $u_{i}$ and $u_{i, j}$ as well as for $V$ and $V_{, j}$ at the boundaries $\Gamma_{l}$ and $\Gamma_{r}$, see Fig. 1 . When $u_{l}$ represents these quantities on the left boundary $\Gamma_{l}$ and $u_{r}$ the quantities on the right boundary $\Gamma_{r}$, the induced periodic boundary conditions take the form

$$
u_{r}\left(x_{j}\right)=u_{l}\left(x_{j}\right) e^{-i k 2 p},
$$

where $k$ is the phase propagation constant of the SAW. ${ }^{21}$ When looking at the case with an alternating electric potential, $k=\pi / 2 p$ is used.

The piezoelectric problem is solved by a plane formulation obtained by setting $S_{i 3}$ and $E_{3}$ as well as $T_{i 3}$ and $D_{3}$ equal to zero. The governing equations (8) are solved simultaneously to find the dependent variables $u_{1}, u_{2}, u_{3}$, and $V$. The model with the PML described above is directly implemented in the partial differential equation application mode of COMSOL MULTIPHYSICS, which is a commercial finite element program. ${ }^{22}$ Second order Lagrange elements are used for all dependent variables.

\section{RESULTS}

In this section, results are presented for the periodic model of the HAR electrodes.

\section{A. Polarization of HAR IDT modes}

One HAR electrode with periodic boundary conditions is considered. The computational domain is illustrated on Fig. 1 where $p=1.4 \mu \mathrm{m}$ is used for definiteness. A harmonic electric potential of $V_{p}=1 \mathrm{~V}$ is applied to excite the device and the resonance frequencies are found by integrating the deflections along the substrate surface for a frequency range. The first six modes are examined. Three of the modes are found to be mainly polarized in the shear-horizontal $(\mathrm{SH})$ direction and the other three modes to be mainly vertically polarized (VP). In Fig. 2, the half phase velocity $f p$ (where $f=\omega / 2 \pi$ is the frequency) of these six modes is plotted ver-

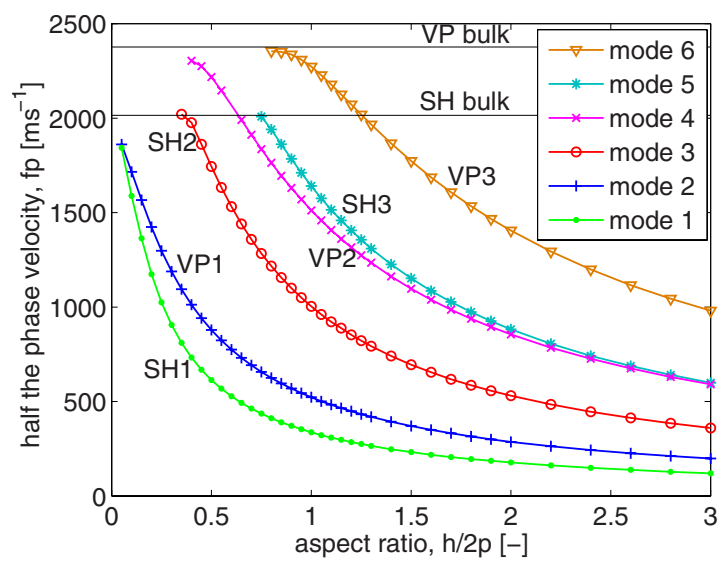

FIG. 2. (Color online) Half of the phase velocity $f p$ as function of $h / 2 p$ for the six first modes for $k p=\pi / 2$. The polarization types are indicated along with the limits for the bulk waves.

sus the aspect ratio of the electrode $h / 2 p$ and the polarization types are indicated. It is seen that the phase velocity is decreasing with increasing aspect ratio for all the modes. The decrease gets bigger for decreasing mode number, and for mode $1 f p$ is decreased up to 15 times. These modes and their polarization as well as the phase velocity dependence of increasing aspect ratio are in fine agreement with the results presented in Ref. 7, where the modes were found by a combined FEM/boundary element method algorithm. The SH and VP bulk wave limits are marked in Fig. 2 as well and the modes do not appear above these limits as they are dissipated to the bulk.

One advantage of using a pure finite element model is that it is easy to plot the displacement fields in the entire structure. Examples are given in Fig. 3 where the deflections $u_{1}, u_{2}$, and $u_{3}$ in the $x_{1^{-}}, x_{2^{-}}$, and $x_{3}$-directions are plotted for the six modes for $h / 2 p=1$. These plots clearly show that all modes of the structure are, in fact, the combination of a vibration in the electrode and of a surface wave in the substrate. The first five modes are fully confined to the surface, whereas mode 6 is leaking into the substrate. The different scaling on the color bars on Fig. 2 indicates the dominant polarization directions for the six modes. In order to clarify these polarizations, a plot for each mode is made in Fig. 4 where the deformations $u_{1}$ and $u_{2}$ in the $x_{1}$-and $x_{2}$-directions are plotted with a scaling factor to emphasize in-plane deformations, and the deflection $u_{3}$ in the $x_{3}$-direction is indicated by the color bar. The plots are focused on the electrode and the regular part of the substrate. The observed modal shapes explain why more and more modes appear in the structure as the aspect ratio increases. When SH-type modes are considered, which are mainly polarized in the $x_{3}$-direction, it is observed that the mode shapes in the electrode in this direction are of increasing order for increasing mode number, such that $\mathrm{SH} 1$ has a mode shape of order $1, \mathrm{SH} 2$ has one of order 2, and SH3 has one of order 3. The same is found for the modes of VP type where VP1 has a mode of order 1, VP2 has a mode of order 2, and VP3 has a mode of order 3 in both the $x_{1}$ - and $x_{2}$-directions. Thus, for larger aspect ratios, more modes can exist as the electrode is allowed to vibrate with modes of higher order. 

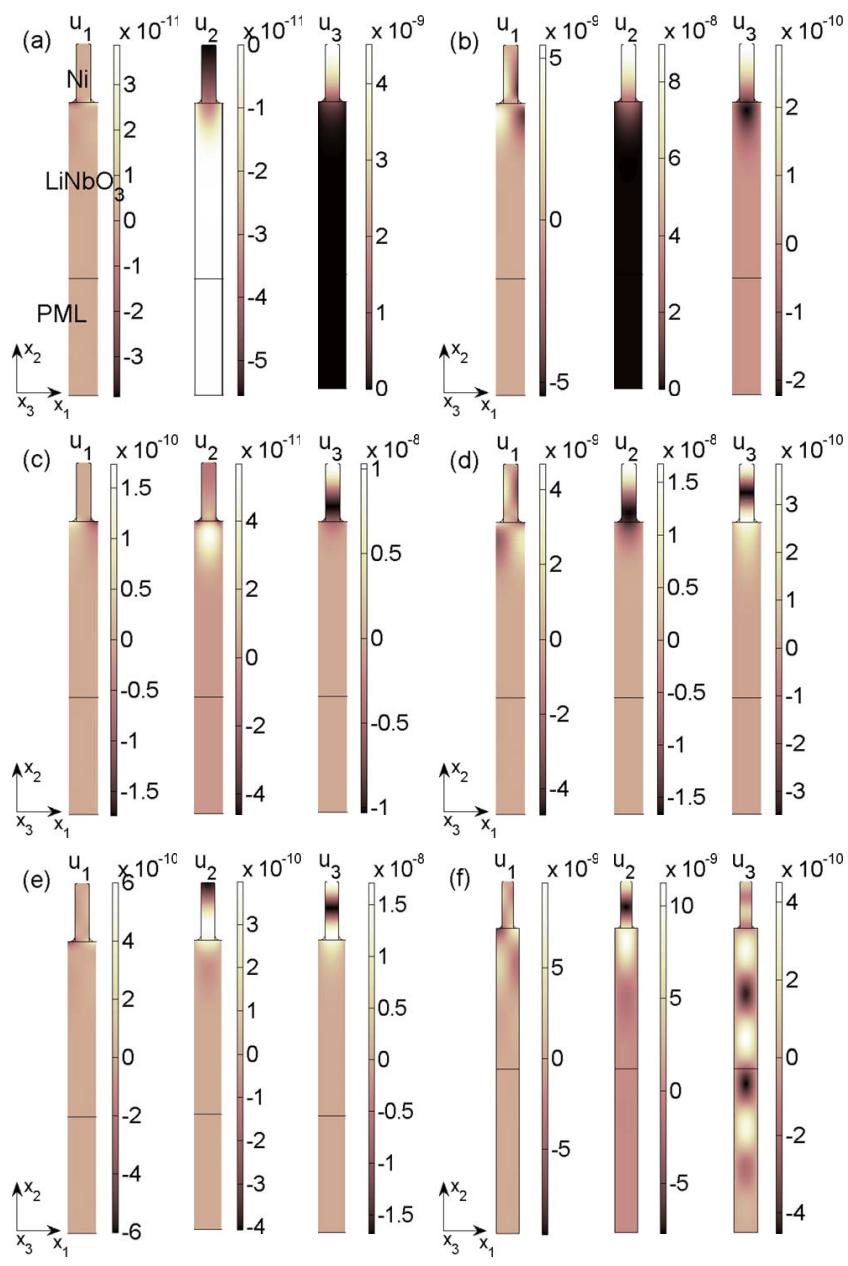

FIG. 3. (Color online) The deflections $u_{1}, u_{2}$, and $u_{3}$ at resonance with $h / 2 p=1$ and $k p=\pi / 2$. All deflections are normalized to $V_{p}=1 \mathrm{~V}$. (a) Mode 1, (b) mode 2, (c) mode 3, (d) mode 4, (e) mode 5, and (f) mode 6.

\section{B. Mechanical energy storage}

To explain the slowing down of the wave velocity, the mechanical energy distribution in the structure is calculated. The mechanical energy is the sum of the stored strain and kinetic energy and the mechanical flux flowing into the PML at the bottom. Expressions for these quantities are given, for instance, in Ref. 20. The fraction of the mechanical energy in the electrode compared to the total mechanical energy in the structure is plotted in Fig. 5. Here it is observed that the

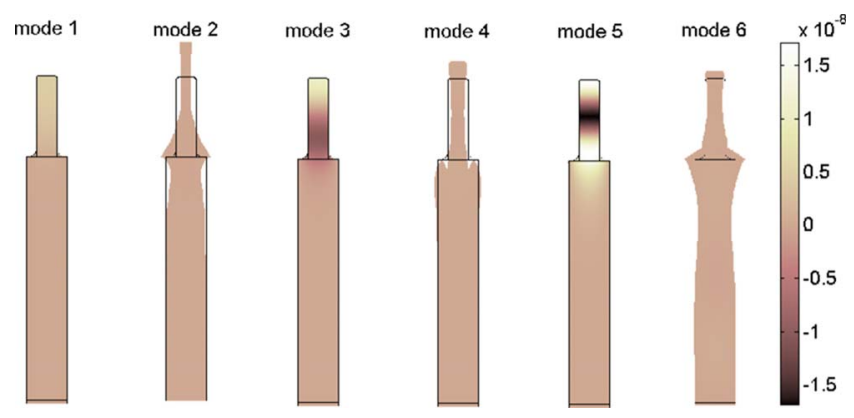

FIG. 4. (Color online) The deflections in the structure for each of the six modes at resonance with $h / 2 p=1$ and $k p=\pi / 2$ normalized to $V_{p}=1 \mathrm{~V}$. The deflections $u_{1}$ and $u_{2}$ are given with an arbitrary scaling factor and $u_{3}$ is given by the color bar for all six modes.

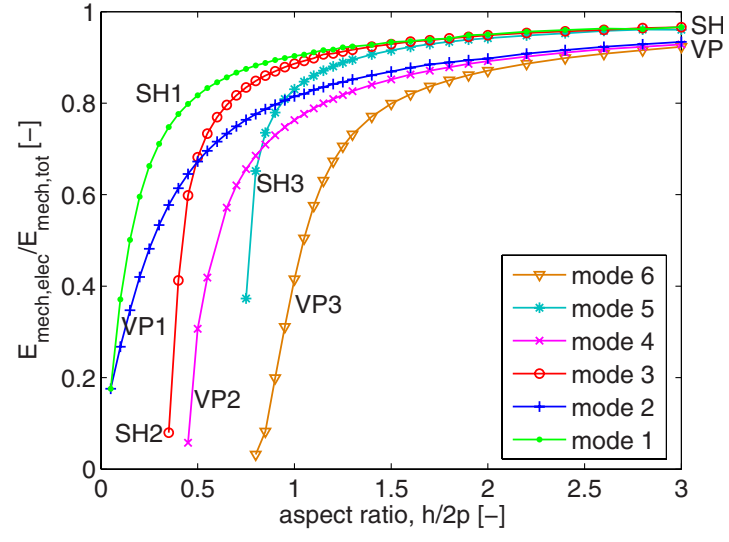

FIG. 5. (Color online) Fraction of mechanical energy in the electrode with respect to the total energy in the structure, as a function of the aspect ratio $h / 2 p$ for the first six modes. The propagation constant is $k p=\pi / 2$. The limit for the SH-type modes is $97 \%$ and the limit for the VP-type modes is $93 \%$.

energy is more confined to the electrode for increasing aspect ratio. The electrode thus acts as a mechanical resonator, which slows down the SAW velocity because of mechanical energy storage. For modes of the same type, the fraction of mechanical energy in the electrode tends to the same value for increasing aspect ratio. The SH types tend to a value around 0.97, which is larger than the limit for the VP types, about 0.93 . The fact that the limits do not reach 1 explains that the wave is still (slightly) propagating or rather coupled from one electrode to the other by surface waves. If the energy was fully trapped then the surface waves would not propagate at all. For increasing aspect ratios the modes are more and more clean cantilever vibrations in the electrode and the only energy left in the substrate is what connects the cantilever modes to the substrate. Modes of the same polarization type deflect in the same direction in the substrate, so the stiffness in the substrate for these modes is the same. This explains why modes of the same type tend to the same energy ratio. The observation that the limit for SH is bigger than the limit for VP is not obvious as SH waves as, e.g., Love waves or leaky-SAW penetrates deeper in the substrate than Rayleigh waves. However, it indicates that the effective mechanical stiffness at the surface is bigger for the VP modes than for the SH modes. This is also confirmed when comparing the rotated stiffness constants in these directions.

\section{Dispersion and group velocity of HAR IDT modes}

A dispersion diagram can be obtained by plotting the frequency as a function of the propagation constant or wave vector. Dispersion tells if the phase velocity is dependent on frequency and is important to know in order to describe propagation in the periodic structure. The normal Rayleigh wave excited by conventional thin electrodes is almost dispersionless and will give a straight line in the diagram just below the straight line corresponding to the slowest shear bulk wave. ${ }^{10}$ The dispersion properties for HAR electrodes are different as illustrated at Fig. 6 where half the phase velocity $f p$ is plotted as function of $k p$ [where the propagation constant $k$ comes from expression (14)] for $h / 2 p=1$. When the wave vector approaches the $X$ point of the Bril- 


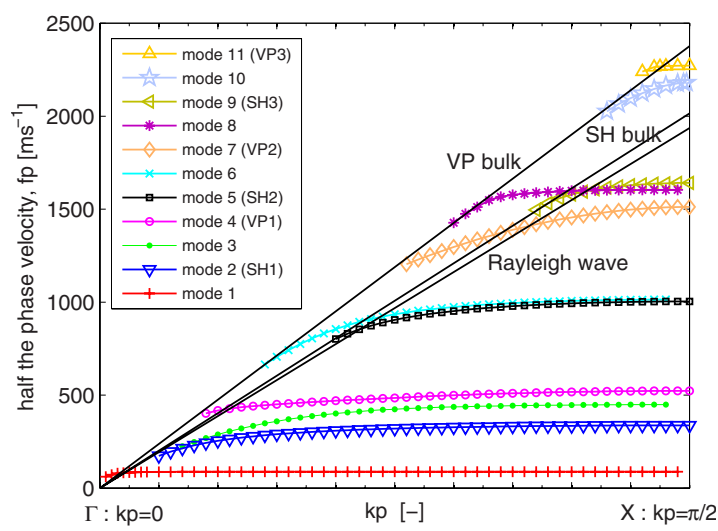

FIG. 6. (Color online) Band diagram for $h / 2 p=1$ obtained by plotting half the phase velocity $f p$ as function of $k p$.

louin zone, all bands become flatter and flatter, which means that the group velocity tends to zero. Then the elastic energy is almost not propagating and is mostly stored in the HAR electrodes. In Fig. 6 the straight lines for the Rayleigh wave as well as the lines for the SH and VP bulk waves are indicated. It is seen that the bands for the SH modes and the VP modes stop at their bulk limits as the waves are dissipated into the bulk material. In the dispersion region following the lowest bulk shear velocity (or Rayleigh SAW) there is an interaction between the Rayleigh SAW and the discrete mechanical modes of the HAR electrodes. When moving away from this line, the interaction between the resonators and SAW reduces (flatband) and the substrate mostly plays the role of a connecting medium for the HAR resonators. The dispersion diagram also shows that there exist 11 modes outside the Brillouin zone limit $X$. The deflections of these additional modes get insignificant when approaching the $X$ point limit and here only the six resonant modes are found in practice to respect the electrical condition and the periodic boundary conditions at the same time. Also in experiments with such structures, only the six resonant modes will be found for alternating electrical potential excitation. ${ }^{7}$ Other values of $k p$ could be selected by using more than two fingers per wavelength in the IDT. For instance, $k p=2 \pi / 3$ and $k p=\pi / 2$ would be obtained with three or four fingers per wavelength, respectively.

The group velocity is calculated from the band diagram by the expression

$$
v_{g}=\frac{\partial \omega}{\partial k}
$$

The results are shown on Fig. 7 where $v_{g}$ is plotted as function of $k p$. The group velocity tends to zero for all 11 modes when the wave vector approaches the point $X$. This means that the velocity of information carried by groups of waves of similar frequencies in the HAR electrodes tends to zero. What is remarkable here is that close to zero group velocity can also be obtained for wave vectors away from the Brillouin zone boundary. This property is more pronounced for modes with smaller orders and is equally true for SH and VP modes.

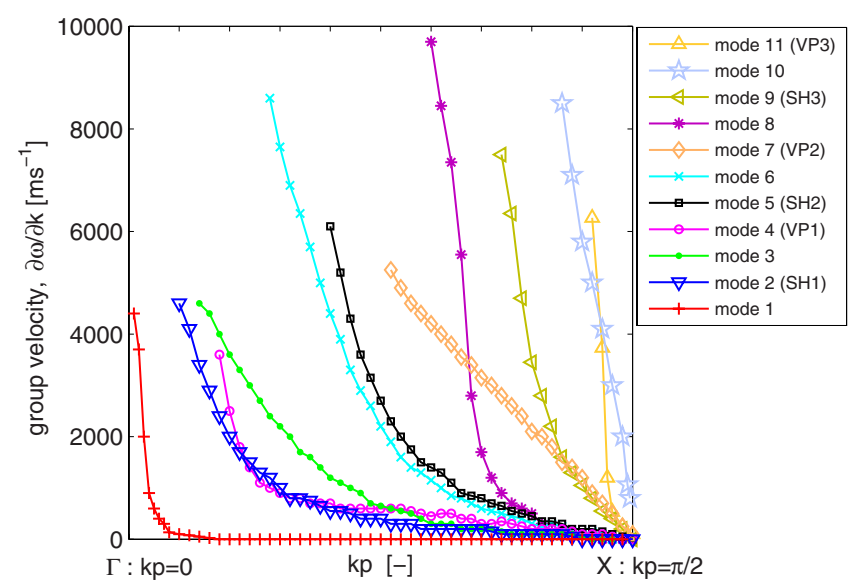

FIG. 7. (Color online) The group velocity $v_{g}=\partial \omega / \partial k$ as function of $k p$ for $h / 2 p=1$.

\section{CONCLUSION AND FURTHER WORK}

This paper elaborates on how to model SAWs generated by high aspect ratio electrodes. It is explained how SAWs propagating in HAR electrode arrays can be computed by employing a 2D model of a piezoelectric, anisotropic material where reflections at the bottom are avoided by a PML. Results are presented for a unit cell with one HAR electrode with periodic boundary conditions. The first six resonant modes are studied and the phase velocity is found to decrease up to 15 times with increasing height. By plotting deflections in all three directions for each mode, it is shown that the movement consists of a combined SAW in the substrate and vibration in the electrode. Three of the modes are mainly polarized in the $\mathrm{SH}$ direction and the three other modes are mainly VP. The deflection plots show that the reason for more and more modes to exist is that for increasing mode number the mode shapes have increasing order. The ratio of mechanical energy confined to the electrodes compared to the total mechanical energy is calculated and increases for increasing electrode height. This indicates that the electrode acts as a mechanical resonator, slowing down the SAW velocity because of mechanical energy storage. The modes of SH type go to the same limit around 0.97 and the modes of VP type go to a limit around 0.93. The band diagram for the structure is calculated and shows strong dispersion for the 11 existing modes where the bands get flatter and flatter when reaching the $X$ point of the first Brillouin zone. Finally, the group velocity is calculated for the 11 modes and shown to tend to zero when reaching the $X$ point for all the modes.

Further work includes the consideration of finite HAR SAW resonators, where mechanical energy storage would be used to avoid leakage outside the transducer. We are also considering using HAR IDTs for efficient acousto-optical interactions in planar optical waveguides.

\section{ACKNOWLEDGMENTS}

This work is supported by the European FP6 research project ePIXnet-European Network of Excellence on Photonic Integrated Components and Circuits. The authors thank the partners from the joint research group of ePIXnet Photo- 
nic Switches and Modulators Based on Surface Acoustic Waves Paulo V. Santos and Markus Beck from Paul-DrudeInstitut für Festkörperelektronik, Berlin, Germany, and Mike van der Poel from Department of Photonics Engineering, Technical University of Denmark, for valuable input related to the model presented.

The authors are grateful to Ole Sigmund and Jakob S. Jensen from the Department of Mechanical Engineering and Martin P. Bendse from the Department of Mathematics, Technical University of Denmark for helpful discussions related to the presented work.

The support from Euro-horcs/ESF European Young Investigator Award (EURYI) through the grant Synthesis and Topology Optimization of Optomechanical Systems as well as from the Danish Center for Scientific Computing (DCSC) is gratefully acknowledged.

${ }^{1}$ R. M. White and F. W. Voltmer, Appl. Phys. Lett. 7, 314 (1965).

${ }^{2}$ K.-Y. Hashimoto, Surface Acoustic Wave Devices in Telecommunications, Modeling and Simulation (Springer, Berlin, 2000).

${ }^{3}$ S. Takahashi, H. Hirano, T. Kodama, F. Miyashiro, B. Suzuki, A. Onoe, T. Adachi, and K. Fujinuma, IEEE Trans. Consum. Electron. CE-24, 337 (1978).

${ }^{4}$ Proceedings of the International Symposium on Surface Acoustic Wave Devices for Mobile Communication, 1992, edited by K. Shibayama and K. Fujinuma (unpublished).

${ }^{5}$ U. Wolff, F. L. Dickert, G. K. Fischauer, W. Greibl, and C. C. W. Ruppel, IEEE Sens. J. 1, 4 (2001).

${ }^{6}$ V. Laude, A. Khelif, T. Pastureaud, and S. Ballandras, J. Appl. Phys. 90,
2492 (2001).

${ }^{7}$ V. Laude, L. Robert, W. Daniau, A. Khelif, and S. Ballandras, Appl. Phys. Lett. 89, 083515 (2006).

${ }^{8}$ T.-T. Wu, Z.-G. Huang, T.-C. Tsai, and T.-C. Wu, Appl. Phys. Lett. 93, 111902 (2008).

${ }^{9}$ Y. Pennec, B. Djafari-Rouhani, H. Larabi, J. O. Vasseur, and A. C. Hladky-Hennion, Phys. Rev. B 78, 104105 (2008).

${ }^{10}$ Y. Zhang, J. Desbois, and L. Boyer, IEEE Trans. Ultrason. Ferroelectr. Freq. Control 40, 183 (1993).

${ }^{11}$ G. Endoh, K. Hashimoto, and M. Yamaguchi, Jpn. J. Appl. Phys., Part 1 34, 2638 (1995).

${ }^{12}$ Y. Fusero, S. Ballandras, J. Desbois, J.-M. Hodé, and P. Ventura, IEEE Trans. Ultrason. Ferroelectr. Freq. Control 49, 805 (2002).

${ }^{13}$ S. Ballandras, M. Wilm, P. F. Edoa, A. Soufyane, V. Laude, W. Steichen, and R. Lardat, J. Appl. Phys. 93, 702 (2003).

${ }^{14}$ J.-P. Berenger, J. Comput. Phys. 114, 185 (1994).

${ }^{15}$ U. Basu and A. Chopra, Comput. Methods Appl. Mech. Eng. 192, 1337 (2003).

${ }^{16}$ M. B. Dühring, Proceedings of 7 th World Congress of Structural and Multidisciplinary Optimization, Seoul, Korea, COEX, 2007 (unpublished).

${ }^{17}$ M. B. Dühring and O. Sigmund, J. Appl. Phys. (in press).

${ }^{18}$ G. Kovacs, M. Anhorn, H. E. Engan, G. Visintini, and C. C. W. Ruppel, Proc.-IEEE Ultrason. Symp. 1, 435 (1990).

${ }^{19}$ D. Royer and E. Dieulesaint, Elastic Waves in Solids, 1st ed. (Springer, New York, 2000).

${ }^{20}$ B. A. Auld, Acoustic Fields and Waves in Solids, 1st ed. (Wiley, New York, 1973).

${ }^{21}$ M. Hofer, R. Lerch, N. Finger, G. Kovacs, J. Schøberl, S. Zaglmayr, and U. Langer, WCCM V, Fifth World Congress on Computational Mechanics, Vienna, Austria, 7-12 July 2002 (unpublished).

${ }^{22}$ COMSOL Reference, Manual for COMSOL 3.3A, COMSOL AB, Stockholm, www.comsol.se. 